\title{
A Cloud based Architecture for Educational Resource Sharing in Ethiopian HEI
}

\author{
Biniyam Wondie \\ M.Sc. in Software Engineering \\ ASTU, Adama, Ethiopia
}

\begin{abstract}
Cloud computing has becomes an adaptable technology in many organization .It represents an important changes in the way information technology is used. Moreover most of the education institutions in the world have become extremely reliant on IT. Universities can take the benefits of cloud based application provided by different services provider and facilitates their The main aim of this research is to design a cloud based architecture to able to share educational resource in Ethiopian higher education institutions.

This study proposes Hybrid cloud platform architecture for sharing of educational resources in the Higher education institutions. For the designed architecture a prototype system was implemented using Microsoft window azure platform. The prototype is very interactive and user friendly which have resource sharing and accessing portal. The developed prototype system was validated using 20 respondents in three higher education staff members and the result is presented in tabular and pie chart form.
\end{abstract}

\section{General Terms}

Cloud Based Architecture for Resource Sharing

\section{Keywords}

Cloud computing, Cloud based architecture, Resource sharing, Software architecture, Higher Education Resource

\section{INTRODUCTION}

Now a day, education is becoming completely associated with the Information and communication technology on the content delivery, communication, storage, share and collaboration. But the use of Internet and Information and Communication Technologies (ICTs) to deliver educational resources is considered mainstream in the 21st century, yet in Higher Education (HE) in developing countries it is often seen as a luxury[5]. This has far reaching effects on teachers, learners and educational institutions in these countries, which often include a lack of basic ICT infrastructure and limited or no support for the training of teachers and learners in the use of digital online information sources. The institutions of higher education must address the studentse requests with respect to IT and the access to institutional networks of wherever and whenever. Consequently, educational institutes recognize the need of adopting new technologies; new methods, instruments and learning techniques in order to satisfy the demand of the age[3].New technologies have revolutionized education, yielding new learning delivery methods. E-learning extended the reach and support of education but relied on tethered computers, and hence was bound by location and time. Mobile learning (M-learning) extends E-learning by breaking these tethers, allowing learning to occur anytime and anywhere [4].
In the past few years the concept of "Cloud Computing (CC)" has emerged as a viable and promising solution to the challenges associated with shrinking IT budgets and escalating IT needs. It has becomes a major points of interest in many fields. Cloud computing has also attracted educational with its potential for delivering economical, securable, reliable, and sharable education services. An increasing number of universities and educational institutions in the USA and UK are adopting cloud computing not only for increased cost savings, but also for improving the efficiency and convenience of educational services [6].

\section{RELATED WORK}

Elamir et al.[42]present a framework naming programming education environment as cloud computing services to teach computer programming. E-learning applications include educational objectives, content production, content delivery technology, assessment and management component. This framework combines the elements from current practices of E-workbook, ELearning and cloud computing technologies. $\mathrm{C}++$ Workbook as cloud service was implemented to validate the proposed framework. Other services are matters of future work such as logging of programming activities, program plagiarism detection, programming workbook and automatic marking [42].

Shaik Saidhbi, et.al. presents [8] Cloud Computing Framework that can be used by Ethiopian Higher Education Institutions, which would enable service delivery much more efficient and effective than the current system.in this research the researcher proposed a framework that will utilize the existing IT infrastructure for institutions which would adapt the framework namely, Ethiopian Universities Hybrid Cloud (EUHC).

Gang Zhao et.al [32] studies the current situation of domestic and foreign cloud computing education, proposed the strategy of constructing information resource libraries in universities under the environment of cloud computing, and studies how to construct the network educational resources platform in institutions of higher learning under the cloud model.

Caifeng Zou et.al [44]The researchers proposed a new model of hybrid cloud computing architecture based on cloud bus. The system is based on local private cloud, combined with one or more types of public cloud. The proposed model of the architecture can accelerate the migration of the existing IT environments to cloud computing environments, reduce the investment, and make full use of IT resources. available, try the font named Computer Modern Roman. On a Macintosh, use the font named Times. Right margins should be justified, not ragged.

Bariah Aljebreen et.al [45] focus on a comprehensive study on scientific literatures in order to find out the opportunity 
and risk of cloud computing in educational fields. This paper also presents the significant information such as the findings, the case studies, related frameworks and supporting also the tools associated to the migration of organizational resources to cloud. Meanwhile, the researcher suggest and design a supplementary strategies when migrating in to the cloud computing service provider especially in kingdom of Saudi Arabia.

\section{A CLOUD BASED ARCHITECTURE FOR EDUCATIONAL RESOURCE SHARING}

Due to different factors, it is true that ICT usage in Ethiopian Higher Education Institutions in its current situation is very primitive, as discussed in previous sections. Harnessing the Cloud Computing technology for delivering different services such as e-learning environment, Class Room Management Systems, Enterprise Resource Planning Systems, researching and similar services will give more flexibilities and dynamic resource utilization which solves the scalability issues. Then the Virtual University Services on top of Cloud Computing layer helps to solve limitations of the current IT service deployment scenario.

So In this study we proposed Cloud Education resources sharing architecture. To get layer and block firstly we analyzed Cloud taxonomy and we got classification related to Cloud Computing such as service type, middleware, and others. However we don't know what relationship between classifications; we reviewed existing cloud computing architecture such as cloud computing reference architecture, IBM cloud computing reference architecture, NIST cloud computing reference architecture and Microsoft cloud academy reference architecture.

\subsection{Conceptual Architecture of Cloud Based Educational Resources Sharing}

Cloud computing solution saves us from having to purchase, maintain and train IT staff on expensive hardware and proprietary software code. With One Network's technology solutions in the cloud, we no longer have to purchase, maintain, update and replace expensive resources. Cloud computing solution saves us from having to purchase, maintain and train IT staff on expensive Hardware and proprietary software code. With One Network's technology solutions in the cloud, we no longer have to purchase, maintain, update and replace expensive resources. Cloud computing solution saves us from having to purchase, maintain and train IT staff on expensive hardware and proprietary software code. With One Network's technology solutions in the cloud, we no longer have to purchase, maintain, update and replace expensive resources.

The user interface is simple, user-friendly and easily understandable, without much graphics and drawings. It will reduce the amount of memory required by the application and reduces the time of development. The original version would be designed with interactive and user-friendly icons and menus.

Software as a Service (SaaS). At the SaaS level, service providers offer consumer or industrial applications directly to individual users and enterprise users. At this level, the following technologies are involved: Web 2.0, Java
Server Page (JSP), C\#, ASP.Net, Microsoft SQL server 2014, Mashup, SOA and multi-tenancy. Education Software as a service for users to manage interactions with Cloud Services in HEIs.

Cloud platform services deliver a computing platform and/or solution stack as a service, often consuming cloud infrastructure and sustaining cloud applications. At the PaaS level, what the service provider's offer is packaged IT capability, or some logical resources, such as databases, file systems, and application operating environment. IaaS, comprised of the physical assets we can see and touch: servers, storage, and networking switches. At the IaaS level, what cloud computing service provider can offer is basic computing and storage capability. Infrastructure as a Service (IaaS) provisions hardware, software, and equipment's to deliver software application environments with a resource usage-based pricing model.

The Management and Integration layer is used to automate the flow of filtered data and information between the horizontal layers. It ensures that processed outputs from one layer to another are contextually related and syntactically correct.

The Security layer ensures the necessary authentication, authorization and auditing for the use of data and services by legitimate users. Further, it ensures secure personalization of end users services based on pre-defined preferences for processing and retrieval of contextual information from a Cloud environment. Each service layer has different levels of security requirement in the cloud based educational resource sharing environment.

\subsection{Architecture for Delivering Educational Resource Sharing Services through Cloud}

This architecture for delivering educational resource sharing services through Cloud based architecture to implement in HEIs.

User layer mainly responsible for checking the track of the authorizations users and its access mode which is selected in the User Interface layer checked them via this module. Users' information is stored in the Users Logs module.

The end-users communicate with the cloud based educational resource sharing using the browser enabled devices such as (handheld computers, notebooks, net-books, ultra-books, tablets and mobile phones) by means of the protocols such as RDP, SSH, Http/Https and LDAP.

In order to use cloud computing services, a device has to ensure secure and reliable network connection. Considering availability, GSM network are the most often used network connection technology in Ethiopia context and also (3G and $4 \mathrm{G}$ ) and wired network connection technology also possible to enable access the services. In terms of reliability, Network bandwidth and latency for Mobile device connection to Internet, best way is using Wi-Fi orWiMax connection.

Cloud that hosts educational resource sharing services can be implemented in hybrid cloud infrastructure in order to use the resources more effectively and also to support the QoS (Quality of Service) objectives such as high availability, performance, reliability, scalability, load balancing and security in the service models. 


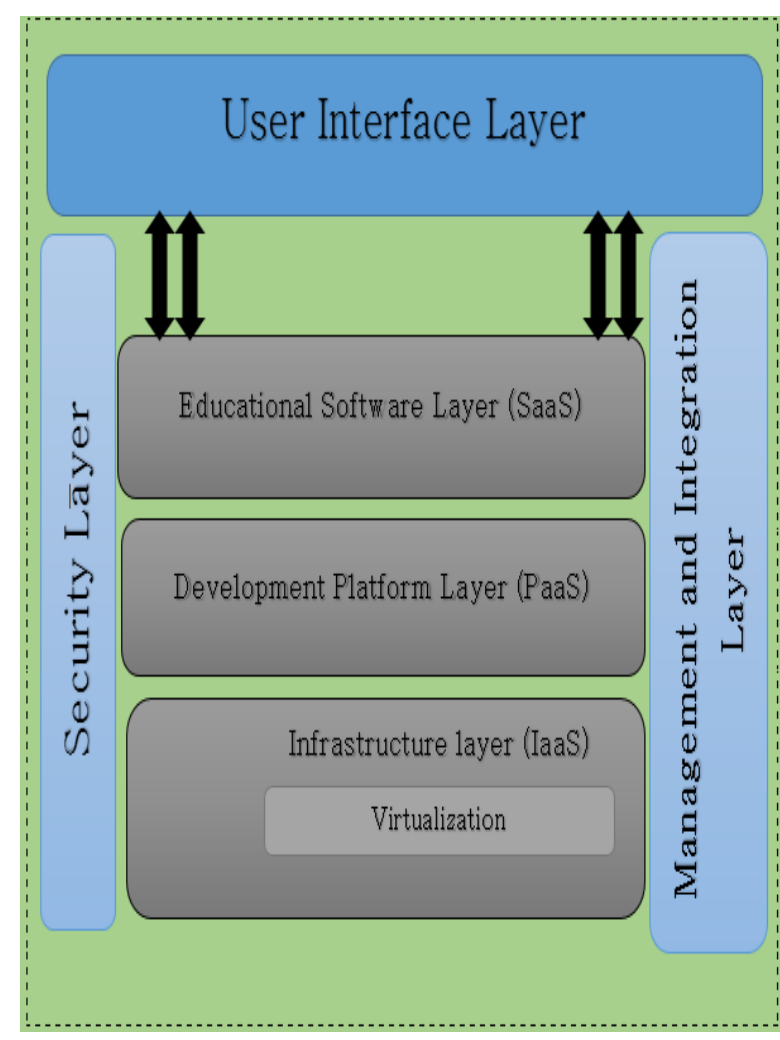

Fig 1: Ethio_HEI Educational Resource Sharing Conceptual Architecture

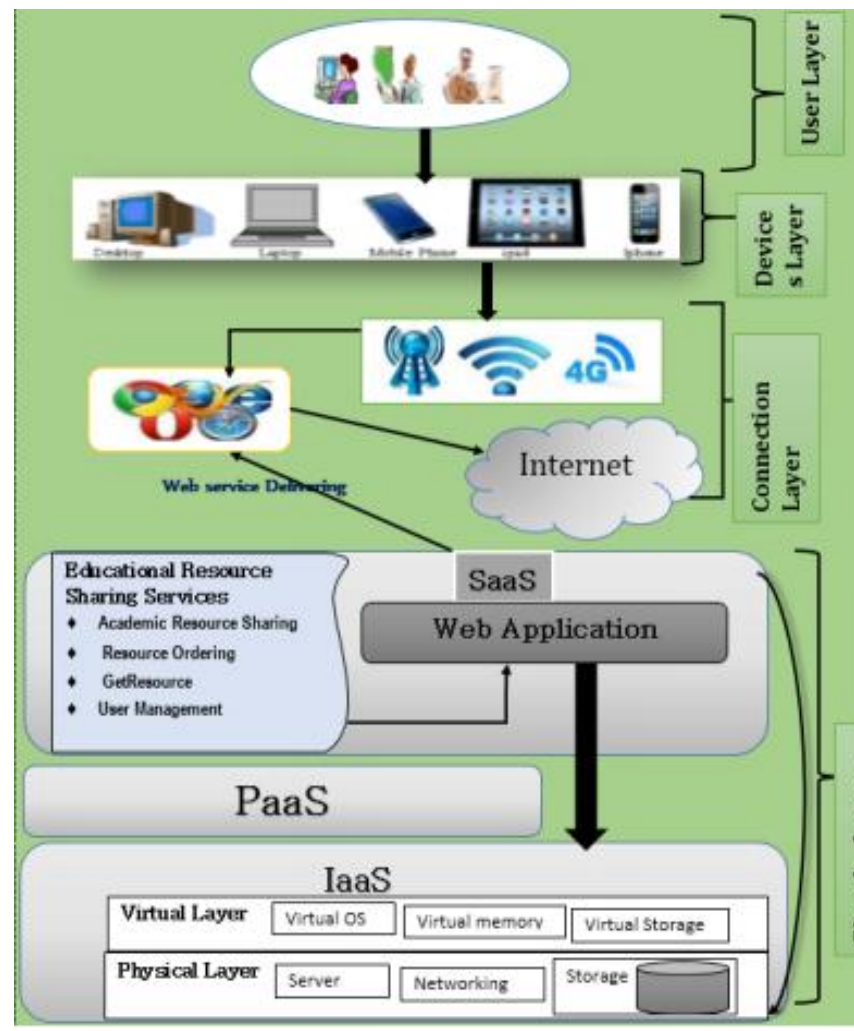

Fig 2: Ethio_HEI Educational Resource Service Delivering Architecture

\section{IMPLEMENTATION OF THE PROPOSED ARCHITECTURE}

The proposed architecture will utilize the existing IT infrastructure for institutions which would adapt the architecture namely, Ethiopian HEI Hybrid Cloud (EHEIHC). In such a situation, the architecture needs to deploy a hybrid cloud model which combines the local (ICT center) infrastructure as a private Cloud with selected public clouds. This Mashup process combines multiple services from different CSPs to serve students and other users from different universities to enhance the teaching-learning and service delivery.

In this research we apply MVC mode, to design the core class of the system, extract classes for the use case, to identify the relationship among the classes and to make the program structure more clear and flexible. Window azure cloud platform selected as the deployment and building application platform, window SQL azure as the storage database as the data storage.

\subsection{Development Environment and Tools}

The development environment for the Ethio_HEI resource sharing cloud based web application is configured as follows:

\section{$>$ Window azure platform(Platform as a Service) \\ $>\quad$ Window azure SDK v2.9 \\ $>$ Internet information service (IIS8) \\ $>\quad$ Visual Studio IDE 2013 \\ $>$ SQL Management Studio 2014}

Microsoft window azure to create and host developed application .So to create the proposed application on window azure platform services: we have used App service; that is the platform services is provided to the user by app service.

The APP services enable developers to focus on their code and reach a stable, highly scalable production state quickly. With APP services, we can develop web app, mobile app, API APP, logic API etc. In window azure platform, there is Visual Studio integration tools that allows for the developer for creating, deploying, and debugging applications in online and at developer local machine.

\subsection{The Developed System Function}

Based on the design architecture a demo web-application developed aiming to the Ethio_HEI resource sharing system to display how the architecture can be used and work in the real world application .Based on the collected data analysis output the system's functions mainly divided into five categories: user management, resource management, Application management, Sharing resource and get resource. Under User management function: there is user registration, user loging, user roles assignation and user authentication and authorization sub function.

Under resource management function: there is resource update, resource delete and add resource type sub function. Under sharing resource function: there is upload resource and edit resource function. Under get resource function: there is download resource and view resource sub function.

So access the web application, the user must use the web address application(Ethio_HEIResourcesSharing.com) and 
then we must login by our account username and password on the web application.

\section{DISCUSSION AND RESULTS}

Ten close ended questions is designed that is further divided in to 5 goal: goal 1 is to assess user interface attractiveness, goal 2 is to evaluate the user friendliness, goal 3 is to measure the performance of the system, goal 4 is to assess the system reliability and goal 5 is to measure the efficiency of the system. For these questionnaires twenty sample size of population is selected: 5 students, $10 \mathrm{HEI}$ teachers and 5 from ministry of education ICT center workers. To evaluate the response of the questionnaires, we used the Likert scale one to five i.e. is 1 (poor), 2(fair), 3(good), 4(very good), 5(excellent).

According to the data gathered from the end user, has very good user interface attractiveness user friendliness performance of the system, system reliability efficiency of the system.

\section{CONCLUSIONS AND FUTURE WORK}

The aim of this work was to identify the particularities of using Cloud based architecture within Higher Education Institutions in Ethiopia. Mainly, we have considered a cloud based architecture for educational resource sharing in EHEIs. And also the study presents a cloud computing resource sharing adoption framework and a roadmap HEIs. we also presents, four platform as a service(PaaS) cloud tools and through analysis, the researcher reached a conclusion Microsoft window azure is a best fit platform for this study. The research shows that hybrid cloud computing is a better choice for deployment in the HEI since it gives the combined benefit of private and public clouds. The proposed hybrid Cloud Computing would be used as a road map for further studies on the topic.

Research in the education fields has much to be examined, but there is not yet a clear definition and standard for such technology. The movement will be rapid after the standardization. Here is a list of some of the future work to be done:

$>$ The proposed architecture should be implemented in each higher education institutions and tested with iterative feedback reflection from the users so that the proposed architecture will be modified based on the feedback before the architecture full implementation for all universities.

$>$ Implement the architecture and measure the framework's effectiveness by having different users. One group of users should use the cloud for every service and the other group of users without using the cloud for their work. Example take two classes who study the same course, the first should use the cloud for course materials, assignments and related course work, and the other class without Cloud based applications.

$>$ Compare the implementation of the architecture on the public Cloud, private Cloud, and hybrid Cloud by highlighting the strengths and weaknesses of each Cloud architecture while considering the performance and security issues. In addition the comparison study may consider the cost, ease of learning, network latency, ease of use, and any other important measurements.

\section{REFERENCES}

[1] B. A. Nan Cenka and Z. A. Hasibuan, "Enhancing educational services using cloud technology," Inf. Commun. Technol. (ICoICT), 2013 Int. Conf., pp. 155160,2013

[2] "Enhancing Cloud based E- Learning using Knowledge Sharing System," vol. 84, no. 9, pp. 26-30, 2013.

[3] C. Study, "The Adoption of Cloud Computing Technology in Higher Education Institutions :

[4] H. Qi and A. Gani, "Research on mobile cloud computing: Review, trend and perspectives," 2012 2nd Int. Conf. Digit. Inf. Commun. Technol. its Appl. DICTAP 2012, pp. 195-202, 2012.

[5] S. Fentahuen and S. Velagapudi, "Cloud Computing Based HPC : A Framework for Ethiopian

[6] Z. S. Alkindi, J. Haynes, and S. Arockiasamy, "A Conceptual Architectural Framework of Cloud Computing for Higher Educational Institutions in the Sultanate of Oman," vol. 6, no. 9, pp. 482-487, 2015.

[7] G. Eugene, "Cloud Computing Models," Massachusetts Intitute Technol., no. January, pp. 1-81, 2013.

[8] M. Hamdaqa and L. Tahvildari, Cloud Computing Uncovered: A Research Landscape, vol. 86. 2012.

[9] E. I. Singh and E. S. Kaur, "Secure Data Sharing in Cloud Computing using Hybrid cloud," pp. 144-150, 1956.

[10] V. H. Pardeshi, "Cloud Computing for Higher Education Institutes: Architecture, Strategy and Recommendations for Effective Adaptation," Procedia Econ. Finance. vol. 11, no. 14, pp. 589-599, 2014.

[11] S. Saidhbi, "A Cloud Computing Framework for Ethiopian Higher Education Institutions," J. Comput. Eng., vol. 66, no. 6, pp. 1-9, 2012.

[12] M. S. Adrees, M. Kamal, and A. Omer, "C LOUD C OMPUTING A RCHITECTURE FOR H IGHER E DUCATION IN T HE T HIRD W ORLD C OUNTRIES (R EPUBLIC OF T HE S UDAN AS M ODEL)," vol. 7, no. 3, pp. 13-24, 2015 